\title{
Low cost metamodel for robust design of periodic nonlinear coupled micro-systems
}

\author{
K. Chikhaoui ${ }^{1,2}$, N. Bouhaddi ${ }^{1}$, N. Kacem ${ }^{1}$, M. Guedri ${ }^{2}$, and M. Soula ${ }^{2}$ \\ ${ }^{1}$ FEMTO-ST Institute, UMR 6174, Department of Applied Mechanics, University of Franche-Comté, UBFC, 24 Rue de \\ l'Epitaphe, 25000 Besançon, France \\ ${ }^{2}$ National High School of Engineers of Tunis (ENSIT), University of Tunis, Tunis, Tunisia
}

\begin{abstract}
To achieve robust design, in presence of uncertainty, nonlinearity and structural periodicity, a metamodel combining the Latin Hypercube Sampling (LHS) method for uncertainty propagation and an enriched Craig-Bampton Component Mode Synthesis approach (CB-CMS) for model reduction is proposed. Its application to predict the time responses of a stochastic periodic nonlinear micro-system proves its efficiency in terms of accuracy and reduction of computational cost.
\end{abstract}

\section{Introduction}

To design large-scale systems incorporating parametric uncertainties with an ever increasing performance, it is inevitable to apply uncertainty quantification and propagation methods to evaluate the influence of the randomness of the structural input parameters on the dynamic behavior of a system. In a probabilistic framework [1], the most frequently-applied stochastic methods are the sample-based methods which consist on a succession of deterministic simulations corresponding to a set of random variables modeling the parametric uncertainties. The Monte Carlo (MC) [2] and the Latin Hypercube Sampling (LHS) [3] methods are the most well-known sample-based methods. Nevertheless, in spite of the flexibility or their implementation, these methods require a great number of samples for a reasonable accuracy and consequently a prohibitive computational cost. Therefore, it is interesting to combine them with reduction methods. In presence of uncertainties and localized nonlinearities, standard reduction methods are no longer efficient since they do not contain any information about these structural modifications. To overcome this issue, the transformation basis used in the Component Mode Synthesis (CMS) approach to reduce each component of the structure can be enriched by taking into account stochastic and nonlinear effects. This advantage enables considering some components including uncertainties and/or localized nonlinearities subject to be reduced independently of the others. Moreover, when considering the case of periodic structures, the CMS approach simplifies the analysis by considering each cell as a component and permits subsequently to attain more important computing time gain. In this work, we focus on the well-known Craig-Bampton CMS (CB-CMS) [4]. In the literature, enriching the Ritz basis with static contribution of neglected eigenvectors was proposed in [5] for accurately evaluating frequency response of modified structures. Masson [6] extends the standard reduction basis by a set of optimized static residual vectors both for direct reductions and CMS approaches. Bouazizi et al. [7] associated the equivalent linearization method with a reduction basis enriched by static residual vectors accounting for parametric modifications and localized nonlinearities. In [8] the CB transformation is enriched with a set of static residual vectors calculated using a priori knowledge of the initial component designs. In [9], to take into account geometrical nonlinearities, the CMS method is enriched by adding the most important modal derivatives, describing second order nonlinear contributions of vibration modes perturbed with the shapes of some others.

Furthermore, combining reduction methods and uncertainty propagation methods was successfully applied in [10] by propagating uncertainties using perturbation techniques at the component level or the global level in a CB-CMS framework. In [11], the CMS is combined with the stochastic spectral finite element method to compute frequency linear structures responses. In [12], the CMS methods are coupled to an adaptive polynomial chaos expansion in order to investigate the frequency transfer functions for large finite element systems with linear and nonlinear stochastic parameters. A robust design optimization is obtained in [13] by combining multi-objective optimization techniques, reduced order method for cheap function evaluations and 
the probabilistic collocation method for uncertainty quantification.

The emphasis of this paper is to develop a metamodel which is robust against uncertainties and nonlinearities and which takes advantage of the structural periodicity. Its efficiency is evaluated in the light of the proposed numerical application which consists on the computation of the time responses of a periodic coupled micro-beams structure containing stochastic parameters and localized nonlinearities. The robustness of the metamodel is evaluated in terms of accuracy and computational time reduction ratio with respect to the reference solutions computed using the LHS method implemented on the full finite element model.

\section{Proposed metamodel}

The sample-based LHS method [3] for uncertainty propagation consists on computing $N$ successive deterministic responses $\left\{y\left(\xi^{(n)}\right), n=1, \ldots, N\right\}$ according to a set of $N$ samples of random variables. The choice of the LHS method is due to its reduced computing time with respect to the very time-consuming MC method. In fact, a great number of samples are typically required in MC to achieve good accuracy while partitioning the variability space into regions of equal probability and picking up one sampling point in each region allow reducing the number of samples needed for the LHS method implementation.

In this paper, the proposed metamodel consists on combining the LHS method and a model reduction method by projecting the responses on the robust enriched basis defined hereafter.

In general case, analyzing the time domain behavior of a nonlinear mechanical system consists on solving the differential equation of motion

$\left\{\begin{array}{l}M \ddot{y}+B \dot{y}+K y+f_{N L}(y, \dot{y})=f_{\text {ext }} \\ y(0)=y_{0}, \dot{y}(0)=\dot{y}_{0} \text { initial conditions }\end{array}\right.$

where $K, M$ and $B$ stand for the stiffness, mass and damping matrices of the system, $f_{N L}$ the nonlinear forces vector and $f_{\text {ext }}$ the exciting forces vector.

Moving from full finite element model to reduced order model requires projecting the time response on a reduction basis, considering the variable transforms $y(t)=T q(t)$ to modal coordinates. Hence, the equation of motion (1) becomes

$$
\begin{aligned}
& T^{T} M T \ddot{q}(t)+T^{T} B T \dot{q}(t)+T^{T} K T q+T^{T} f_{N L}(q, T \dot{q})= \\
& T^{T} f_{\text {ext }}=
\end{aligned}
$$

Solving the equation (2) in the time domain is done using the nonlinear Newmark time integration scheme and the
Newton-Raphson prediction-correction algorithm [14, $15]$.

In deterministic linear case, the reduced model is obtained using standard CB-CMS of the linear system. In this case, the $\mathrm{CB}$ transformation (CBT) is defined, for a component $k$, as

$y^{k}=\left\{\begin{array}{l}y_{j} \\ y_{i}\end{array}\right\}^{k}=\left[\begin{array}{cc}I_{j j} & 0 \\ \psi_{i j} & \varphi_{i}\end{array}\right]^{k}\left\{\begin{array}{l}q_{j} \\ q_{c}\end{array}\right\}^{k}=T_{C B}{ }^{k} q^{k}$

where $y_{i}^{k}$ are the interior coordinates reduced to modal coordinates $q_{c}{ }^{k}, y_{j}{ }^{k}$ are the junction coordinates, $\psi_{i j}{ }^{k}=-\left(K_{i i}{ }^{k}\right)^{-1} K_{i j}{ }^{k}$ is the static subbasis which contains the constraints modes, $I_{j j}$ is the identity matrix, and $\varphi_{i}{ }^{k}$ is the truncated basis containing the first $n_{r}$ normal modes of the corresponding component.

Nevertheless, a standard CBT basis does not contain any information about uncertainties and nonlinearities. Therefore, adding complementary subbases $\Delta T^{k}$ to enrich $T_{C B}{ }^{k}$ is necessary. The $\Delta T^{k}$ subbases contain a well selected static residual vectors calculated according to the type of enrichment. Once the complementary subbases $\Delta T_{N L}{ }^{k}, \Delta T_{S}{ }^{k}$ and $\Delta T_{E}{ }^{k}$, corresponding respectively to the nonlinear, stochastic and external excitation enrichments are generated, the enriched CBT (ECBT) $T_{E C B}{ }^{k}$, for each component $k$, takes the form

$$
\begin{aligned}
& T_{E C B}{ }^{k}=\left[\begin{array}{lllllll}
T_{C B} & \vdots & \Delta T_{N L} & \vdots & \Delta T_{S} & \vdots & \Delta T_{E}
\end{array}\right]^{k} \\
& =\left[\begin{array}{cccccccc}
I_{j j} & 0 & & 0 & & 0 & & 0 \\
\psi_{i j} & \varphi_{i} & & \Delta T_{N L} & & \Delta T_{S} & & \Delta T_{E}
\end{array}\right]^{k}
\end{aligned}
$$

This transformation matrix contains $n_{j}^{k}$ junction dofs, $n_{r}^{k}$ retained normal modes and $n_{e}^{k}$ enriching static residual vectors.

\section{Numerical simulations}

The proposed stochastic periodic nonlinear micro-system, Figure 1, is composed of twenty identical coupled microbeams submitted to transversal vibration. The discretization of each micro-beam into 20 elements leads to a FEM of 800 degrees of freedom (dofs). The microbeams, which geometrical and mechanical parameters are listed in Table1, are coupled using twenty localized linear dampers and linear and nonlinear springs. Each beam $i$ is submitted to a localized excitation force $P_{f i}(N)=$ $10^{-6} \cos \left(2 \pi f_{1} t\right)$ where $f_{1}=565.53 \times 10^{3} \mathrm{~Hz}$ is the first eigenfrequency.

The time responses evaluation is done in the time interval $\left[0-5 \times 10^{-5} \mathrm{~s}\right]$, divided into steps of $10^{-8} s$, in which the stationary regime is already attained. 


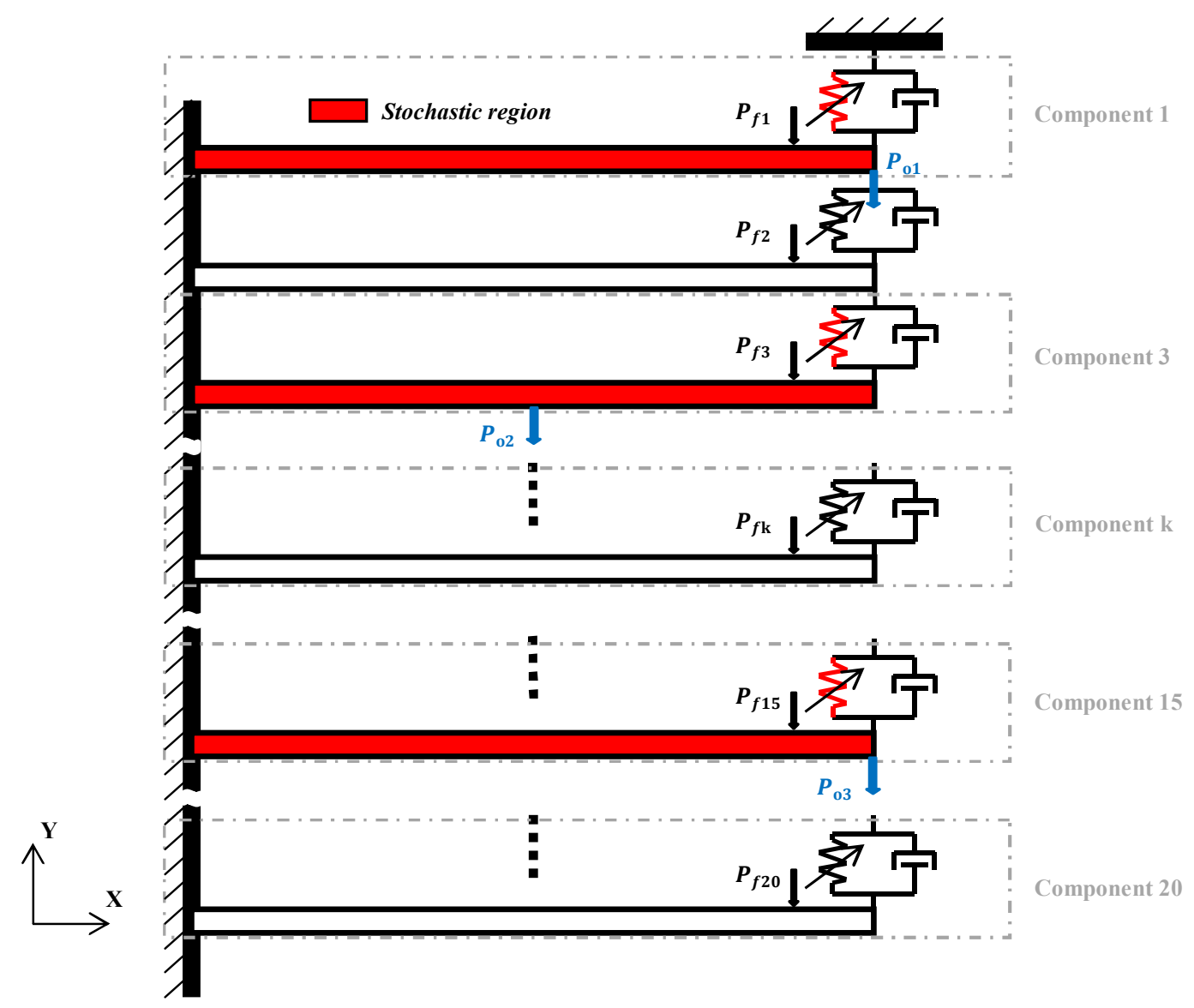

Fig. 1. Model of periodic nonlinear coupled micro-system.

Table 1. Geometrical and mechanical parameters of the micro-system.

\begin{tabular}{|c|c|c|c|c|c|c|c|}
\hline $\begin{array}{c}b \\
(\mu m)\end{array}$ & $\begin{array}{c}h \\
(\mu m)\end{array}$ & $\begin{array}{c}L_{b} \\
(\mu m)\end{array}$ & $\begin{array}{c}E_{0} \\
(G P a)\end{array}$ & $\begin{array}{c}\rho_{0} \\
\left(k g . m^{-3}\right)\end{array}$ & $\begin{array}{c}c \\
\left(N . s . m^{-1}\right)\end{array}$ & $\begin{array}{c}k_{0} \\
\left(N . m^{-1}\right)\end{array}$ & $\begin{array}{c}k_{N L} \\
\left(N . m^{-3}\right)\end{array}$ \\
\hline 3 & 1 & 50 & 169 & 2330 & $10^{-5}$ & 10 & $10^{14}$ \\
\hline
\end{tabular}

Being in the case of a periodic structure, to apply the CB-CMS method, we propose to consider each set of coupling elements (localized linear damper, linear and nonlinear springs) and a micro-beam as a component (Fig. 1). Three stochastic zones are considered including the first, the third and the fifteenth components. In these zones, the Young modulus of the micro-beam and the linear coupling stiffness are supposed to be the uncertain parameters such as

$p=p_{0}\left(1+\delta_{p} \xi_{p}\right)$

where $p$ refers to the parameter and $\xi_{p}$ are two types of random variables of uniform and lognormal probability distributions modeling respectively the Young Modulus and the linear stiffness uncertainties. $\delta_{p}=10 \%$ are the considered dispersions.

The (1-MAC) matrix, Figure 2, shows the effect of the uncertainties of the associated linear structure (MAC for Modal Assurance Criterion). This matrix compares the normal modes of the deterministic model with the mean of stochastic modes computed for each random variable of the stochastic model computed using the LHS method on the full finite element model. If $(1-\mathrm{MAC})=0$, the compared modes are consistent. If $(1-\mathrm{MAC})=1$, no correlation between these modes occurs.

The responses are presented in terms of velocities in order to illustrate the nonlinear effect. Figures 3-5 show the time responses of three observation degrees of freedom (dofs) obtained when applying the proposed metamodel (LHS-ECBT) and the reference LHS method implemented on the full finite element model (LHSREF).

To compare the obtained results in terms of accuracy and time consuming, the Central Processing Unit (CPU) time and the time indicators $E, T$ and $D^{2}$ (Table 2) are calculated. These time indicators, also called energy criteria (Hemez and Doebling, 2003), are calculated using the time moments

$\mathcal{M}_{i}=\int_{-\infty}^{+\infty}\left(t-t_{s}\right)^{i} y(t)^{2} d t$

where $y(t)$ is the time response, $i$ the order of the moment and $t_{s}$ the temporal shift chosen in our case as 
$t_{s}=0$. In fact, $E=\mathcal{M}_{0}$ is the total energy of the response, $T=\mathcal{M}_{1} / \mathcal{M}_{0}$ the central time and $D^{2}=$ $\mathcal{M}_{2} / \mathcal{M}_{0}-\left(\mathcal{M}_{1} / \mathcal{M}_{0}\right)^{2}$ the root mean square duration, $E$ permits to evaluate the accuracy of the response in term of amplitude while $T$ and $D^{2}$ allow it in term of periodicity.

As shown in Table 2, a model reduction ratio of $90 \%$ (reduced model of 82 dofs) and a computational time gain of $55 \%$ can be achieved when implementing the metamodel compared to the reference model. The accuracy errors are less than $2.5 \%$ and $0.14 \%$ in terms of amplitude and periodicity respectively. To reduce these errors, other vectors must be added in the transformation basis.

It is important to note that taking into account the periodicity of the structure permits to simplify the implementation of the CB-CMS method by taking the unit cell as a component.

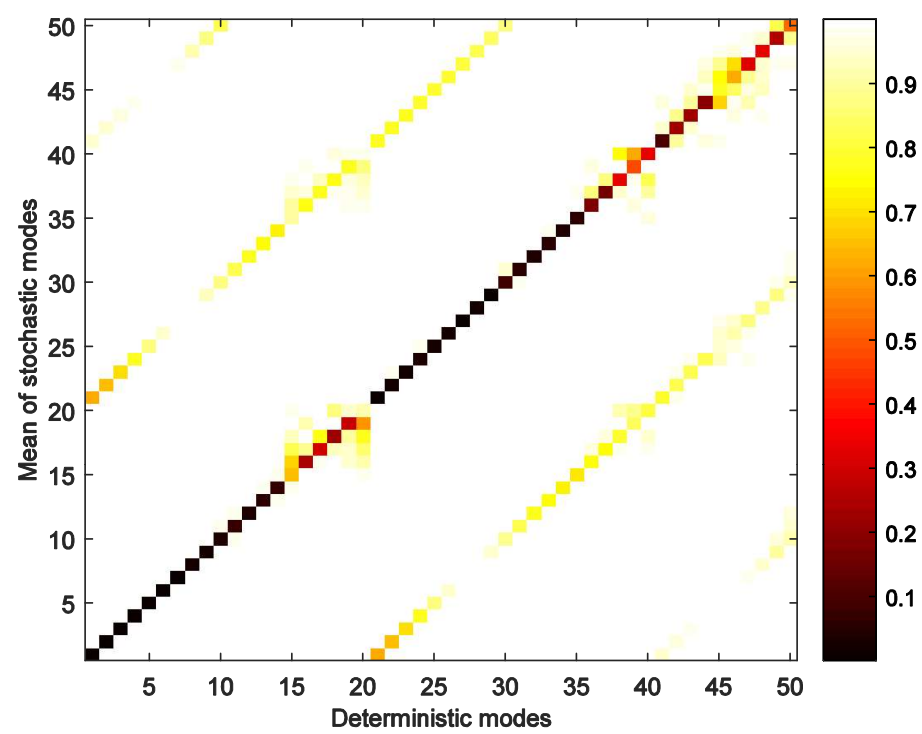

Fig. 2. (1-MAC) matrix illustrating the comparison of deterministic normal modes and the means of the stochastic modes.
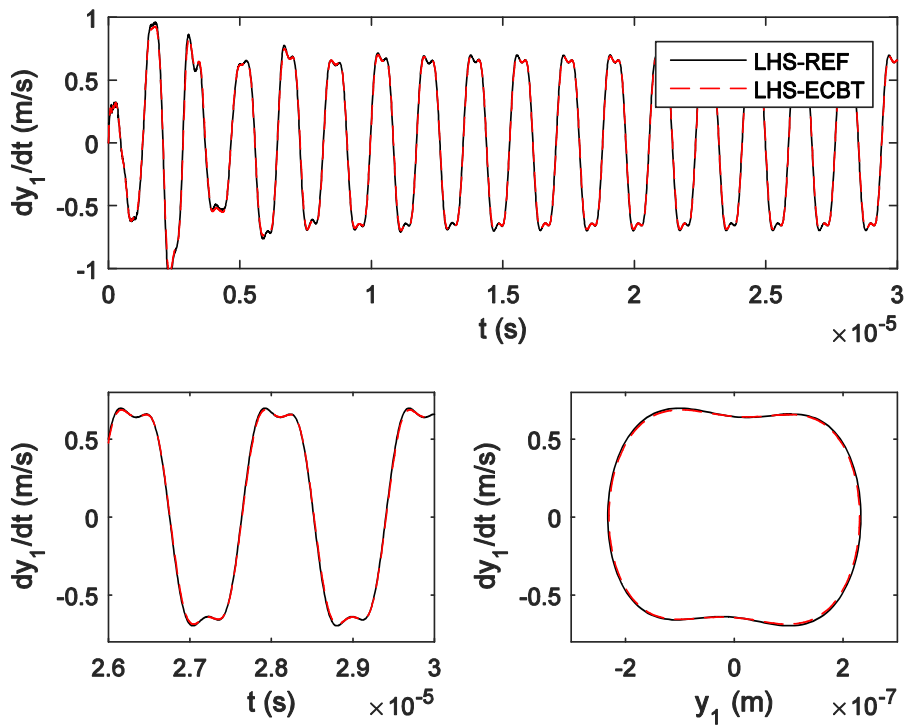

Fig. 3. Mean of the stochastic velocities and phase diagrams, at the observation dofs $P o_{1}$, computed using LHS-REF and LHSECBT methods. 

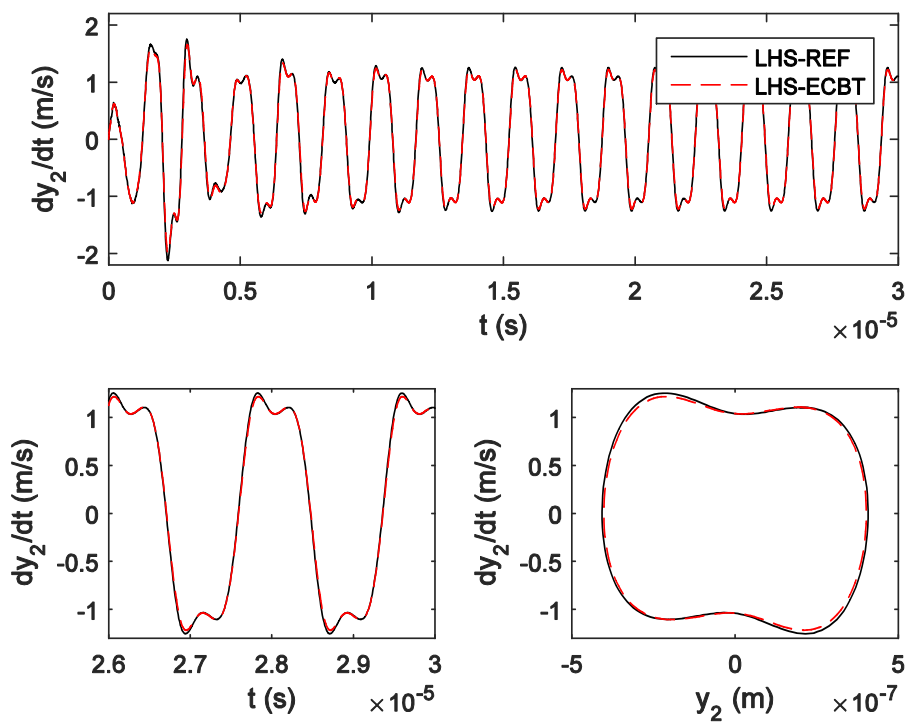

Fig. 4. Mean of the stochastic velocities and phase diagrams, at the observation dofs $\mathrm{Po}_{2}$, computed using LHS-REF and LHSECBT methods.
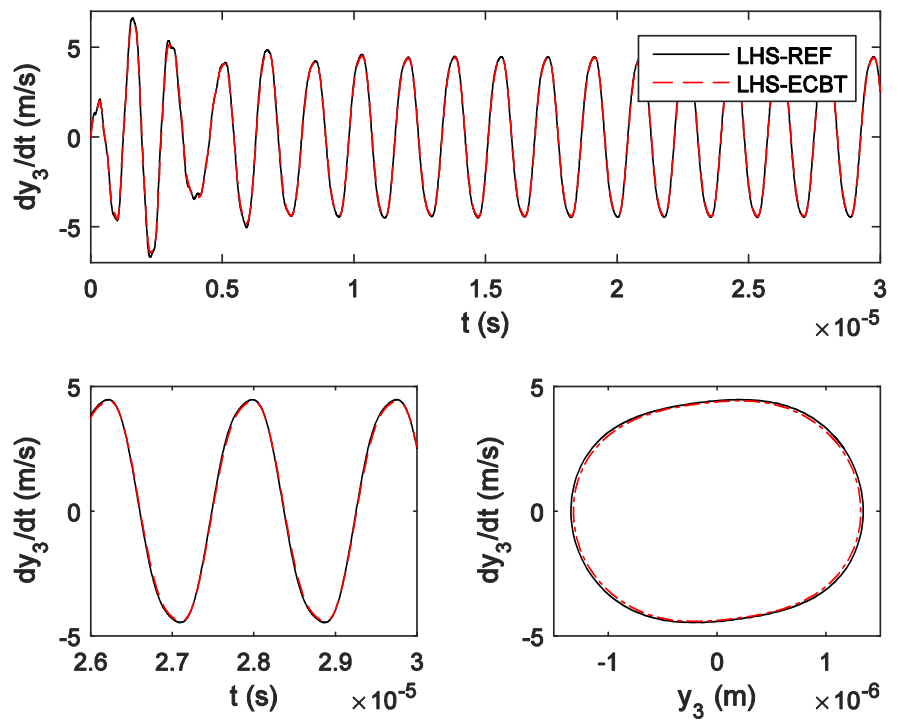

Fig. 5. Mean of the stochastic velocities and phase diagrams, at the observation dofs $\mathrm{Po}_{3}$, computed using LHS-REF and LHSECBT methods.

Table 2. Model size, time indicators and CPU time.

\begin{tabular}{|c|c|c|c|c|c|}
\hline \multirow{2}{*}{ Methods } & \multirow{2}{*}{$\begin{array}{c}\text { Model size } \\
\text { (dofs) }\end{array}$} & \multicolumn{2}{|c|}{ Time indicators (error \%) } & \multirow{2}{*}{ CPU (\%) } \\
\cline { 3 - 5 } & & $\mathbf{E}$ & $\mathbf{T}$ & $\mathbf{D}^{\mathbf{2}}$ & \\
\hline LHS-REF & 800 & - & - & - & 100 \\
\hline LHS-ECBT & 82 & 2.50 & 0.07 & 0.14 & 45.1 \\
\hline
\end{tabular}




\section{Conclusion}

This paper proposed a metamodel combining the LHS method for uncertainty propagation and the CB-CMS for model reduction. In the light of the results of the presented application, the proposed metamodel permits to approximate the structure behavior with a reasonable computational cost and without a significant loss of accuracy, with respect to the reference method and so proves to be a powerful tool for robust design of periodic nonlinear micro-systems.

\section{References}

1. C. Soize, Eur J Comp Mech 19: 25-40 (2009)

2. R. -Y. Rubinstein, John Wiley \& Sons (1981)

3. J. C. Helton, F. J. Davis, Reliab Eng Sys Safe 81: 2369, (2003)

4. Jr. R. R. Craig, M.C.C. Bampton, AIAA J 6 (7): 1303-1319, (1968)
5. E. Balmès, Mech Syst Signal Pr 10 (4): 381-394, (1996)

6. G. Masson, B. Ait Brik, S. Cogan, N. Bouhaddi, J Sound Vib 296 (4-5): 845-860, (2006)

7. M. L. Bouazizi, M. Guedri, N. Bouhaddi, Mech Syst Signal Pr 20: 131-157, (2006)

8. D. A. Perdahcioglu, M. H. M. Ellenbroek, A. De Boer, ICSV16, July 5-9, Krakow, Poland, (2009)

9. F. Wenneker, P. Tiso, In M. Allen et al. (Eds) Dynamics of Coupled Structures 1: 157-165, (2014)

10. L. Hinke, F. Dohnal, B. R. Mace, T. P. Waters, N. S. Ferguson, J Sound Vib 324: 161-178, (2009)

11. M. Guedri, N. Bouhaddi, R. Majed, J Sound Vib 297: 123-45, (2006)

12. D. Sarsri, L. Azrar, A. Jebbouri, A. El Hami, Comput Struct 89: 346-356, (2011)

13. R. S. Motta, S. M. B. Afonso, P. B. Lyra, R. B. Willmersdorf, Eng Comput, 32 (2): 258-288, (2015)

14. M. Gérardin, D. Rixen, John Wiley \& Sons, (1997)

15. S. Krenk, Cambridge University Press, (2009) 